\title{
Correction: Early use of combined exogenous surfactant and inhaled nitric oxide reduces treatment failure in persistent pulmonary hypertension of the newborn: a randomized controlled trial
}

\author{
Alvaro González (D) Aldo Bancalari · Waldo Osorio · Matías Luco • Agustina González • Héctor Pérez · Javier Kattan
}

Published online: 7 September 2020

(c) The Author(s), under exclusive licence to Springer Nature America, Inc. 2020

Correction to: Journal of Perinatology

https://doi.org/10.1038/s41372-020-00777-x
This article was originally published without the trial registration number ISRCTN13727958. This has now been included below the abstract. 